\title{
Evaluation of guidelines for emergency triage assessment and treatment in developing countries
}

Giorgio Tamburlini, Simona Di Mario, Ruben Schindler Maggi, Jose Nivaldo Vilarim, Sandy Gove

\begin{abstract}
Aim-To evaluate performance of a simplified algorithm and treatment instructions for emergency triage assessment and treatment (ETAT) of children presenting to hospital in developing countries.

Methods-All infants aged 7 days to 5 years presenting to an accident and emergency department were simultaneously triaged and assessed by a nurse and a senior paediatrician. Nurse ETAT assessment was compared to standard emergency advanced paediatric life support (APLS) assessment by the paediatrician. Sensitivity, specificity, and predictive values were calculated and appropriateness of nurse treatments was evaluated.

Results-The ETAT algorithm as used by nurses identified $731 / 3837$ patients $(19.05 \%)$; 98 patients $(2.6 \%)$ were classified as needing emergency treatment and $633(16.5 \%)$ as needing priority assessment. Sensitivity was $96.7 \%$ with respect to APLS assessment, $91.7 \%$ with respect to all patients given priority by the paediatrician, and $85.7 \%$ with respect to patients ultimately admitted. Specificity was $90.6 \%, 91.0 \%$, and $85.2 \%$, respectively. Nurse administered treatment was appropriate in $94 / 102(92.2 \%)$ emergency conditions.
\end{abstract}

Unit for Health Services Research and International Health and Department of Paediatrics, IRCCS Burlo Garofolo, via dell'Istria 65/1, 34137, Trieste, Italy G Tamburlini

S Di Mario

Instituto Materno

Infantil de

Pernambuco, Recife,

Brazil

R S Maggi

J N Vilarim

Berkeley School of Public Health, Division of Public

Health Biology and Epidemiology,

Berkeley, California, USA

$S$ Gove

Correspondence to:

Dr Tamburlini

email: tamburli@

burlo.trieste.it

Accepted 19 July 1999
The main objective of the study was to evaluate the performance of the emergency triage assessment and treatment (ETAT) algo- rithm, as used by nurses for identifying children who need priority assessment and treatment from those attending the hospital emergency service.

Secondary objectives were: to evaluate the appropriateness of emergency treatment administered by nurses; to evaluate the agreement between nurses and experienced paediatricians in the recognition of the risk signs included in the algorithm; to characterise the proportion of children attending the accident and emergency department who have the various ETAT signs indicating the need for emergency treatment or priority assessment; and to define the outcome of these children in terms of hospitalisation and early mortality rates.

Study population and methods

STUDY SITE

The study was carried out at the emergency department of the Instituto Materno-Infantil de Pernambuco (IMIP), a 600 bed mother and child hospital in Recife, Brazil, mainly serving the underprivileged population of the Recife area. An average of 180 children attend the emergency department each day. Before the study there were no specific triage guidelines, and nurses did not provide any emergency treatment except on doctors' orders. The climate in Recife is tropical, hot, and humid throughout the year, with a "wet" and relatively cooler season from March to September. The epidemiology of childhood diseases is typical of a developing country, with pneumonia, diarrhoea, sepsis, meningitis, and severe malnutrition being the main causes of death. Malaria is uncommon.

SELECTION AND TRAINING OF HEALTH PROFESSIONALS PARTICIPATING IN THE STUDY Six registered nurses and two senior paediatricians, all working at the IMIP, were selected to participate in the study. The two paediatricians had received recent standard training in advanced paediatric life support (APLS) in the UK. ${ }^{3}$ The six nurses, all with three to four years of specific nurse training and previous experience in paediatric inpatient care, received ETAT training in the IMIP during the week before the study began. This comprised 10 hours of theoretical and 10 hours of clinical sessions. The former included presentations, drills, and case studies. During the latter participants were asked to identify specific signs, to assess patients, and if necessary administer treatment to real cases. The clinical sessions were carried out at the emergency department and at the paediatric ward of IMIP. 
STUDY PROTOCOL

All children aged more than 7 days and less than 5 years who were brought to the emergency department during study hours were simultaneously triaged and assessed by one of the six trained nurses and by one of the two senior paediatricians participating in the study. The study period covered 10 weeks (14 July to 30 September 1997) with study hours from $07: 00$ to $13: 00$ and from $14: 00$ to $18: 00$, Monday to Friday, and from 19:00 to midnight twice weekly, for a total of 60 hours per week. One of the six nurses and one of the two senior paediatricians were in charge at the emergency department during the study hours.

Nurses used the ETAT guidelines and paediatricians the APLS guidelines. To avoid any possible contamination of the nurses' assessments by the doctors, each nurse made explicit the results of her assessment and treatment choices before the doctor did.

To children who were identified as presenting with any of the emergency conditions according to ETAT (group 1) the nurse administered the recommended emergency treatment, then asked the doctor to proceed with further assessment and treatment.

For patients identified by a nurse as showing priority signs (group 2) the nurse asked the paediatrician to proceed with priority assessment and treatment.

The paediatrician, besides assessing the patients according to APLS guidelines, checked the nurse's assessment according to ETAT guidelines and supervised the treatment administered by the nurse in group 1 patients. The paediatrician intervened when the treatment chosen was inappropriate or unnecessarily delayed. The paediatrician also completed a full medical assessment for all children identified by either ETAT or APLS as priority patients and administered any indicated treatment.

Any other child identified by nurses as needing priority assessment, but who did not meet ETAT criteria, was recorded. Similarly, any patient that paediatricians identified as needing priority assessment and management, but did not have positive APLS signs, was recorded. When the assessment and/or the treatment made by the nurse were not appropriate, the reasons for this were systematically recorded. All cases were followed up and data on outcomes (admission, final diagnosis, treatment, death within 24 hours from admission) were also collected. The age and sex of all the patients seen at the emergency department during the study period were recorded to provide the denominator of the study population.

All data were recorded by an additional member of the research team, a paediatrician also specifically trained in ETAT, who was present during the whole study period at the emergency department.

REFERENCE STANDARDS AND DATA ANALYSIS For the purpose of the study, the reference standards that were used for evaluating the performance of ETAT as used by nurses as a screening tool were: (a) patients with a positive sign on APLS assessment made by experienced paediatricians (the APLS assessment and differences between ETAT and APLS have been described elsewhere $)^{23}$; (b) all priority cases as identified by experienced paediatricians (all APLS positive cases plus any other case identified by the paediatrician as needing priority management); (c) all admitted patients.

Sensitivity, specificity, and predictive values with $95 \%$ exact binomial confidence intervals (CIs) for the overall ETAT algorithm as applied by nurses were calculated with respect to the above reference standards.

The appropriateness of treatment administered by nurses to patients presenting with emergency conditions (group 1 patients) was evaluated by the paediatrician with respect to the content of treatment (drugs and dosages), the mode of administration, and the time to treatment. Since all emergency treatments were supposed to be administered immediately, any unnecessary delay prompted the intervention of the paediatrician.

Clinical agreement between nurses and doctors in identifying ETAT emergency and priority signs was calculated by the means of $\kappa$ test on all signs included in the ETAT algorithm, with the exception of age less than 2 months and urgent referral note (these two criteria being self evident), and of visible respiratory distress (APLS included auscultation so that uncontaminated assessment by doctors based on visible respiratory distress only was not possible).

The data were analysed using Epi Info version 6.0 (Centers for Disease Control, Atlanta, Georgia, USA) computer software. The study was approved by the IMIP ethics committee.

\section{Results}

PROPORTION AND OUTCOME OF CHILDREN WITH SIGNS INDICATING A NEED FOR EMERGENCY TREATMENT OR PRIORITY ASSESSMENT A total of 3837 infants and children aged 7 days to 5 years attended the emergency department of IMIP during the study period. Three hundred and eighty six $(10.1 \%)$ were less than 2 months old, and 1368 (35.6\%) less than 1 year old. The male to female ratio was 1.27 .

Overall, the ETAT algorithm, as used by the nurses, identified 731 patients $(19.1 \%$ of all patients attending the emergency department) as having one or more emergency or priority signs; $98(2.6 \%)$ had one or more emergency conditions indicating a need for emergency treatment (group 1). The remaining 633 cases $(16.5 \%)$ had signs indicating a need for priority assessment and treatment (group 2). There were 56 additional children with priority signs who also had emergency signs and were classified as group 1 .

The nurses identified 24 further patients with signs not included in the ETAT algorithm but which they interpreted as obvious priority signs (trauma, obvious surgical cases such as strangulated inguinal hernia, severe skin or eye 
Table 1 Distribution, admission, and mortality rates of classifications and signs of ETAT algorithm

\begin{tabular}{|c|c|c|c|c|c|c|}
\hline ETAT classification and signs & Patients attended & $\begin{array}{l}\% \text { of all patients attended at } \\
\text { emergency department }\end{array}$ & $\begin{array}{l}\text { Patients } \\
\text { admitted }\end{array}$ & $\begin{array}{l}\% \text { of all admitted } \\
\text { patients }\end{array}$ & Deaths* & $\%$ of all deaths \\
\hline ETAT group 1 (emergency condition) & 98 & 2.55 & 85 & 36.9 & 10 & 71.4 \\
\hline Severe respiratory distress & 57 & 1.48 & 44 & 19.1 & 10 & 71.4 \\
\hline Shock & 4 & 0.13 & 2 & 0.86 & 4 & 28.5 \\
\hline Coma/convulsions & 14 & 0.36 & 10 & 4.35 & 3 & 21.4 \\
\hline Severe dehydration & 30 & 0.73 & 23 & 10.0 & 1 & 7.1 \\
\hline ETAT group 2 (priority signs) & $689(633) \dagger$ & $17.9(16.5) \dagger$ & 112 & 48.7 & 4 & 28.5 \\
\hline Non-severe respiratory distress & 213 & 5.6 & 59 & 25.6 & - & - \\
\hline Visible severe wasting & 34 & 0.86 & 27 & 11.7 & 1 & 7.1 \\
\hline Severe palmar pallor & 8 & 0.21 & 7 & 3.0 & 1 & 7.1 \\
\hline Oedema of both feet & 19 & 0.49 & 15 & 6.5 & - & - \\
\hline Lethargy & 8 & 0.21 & 5 & 2.2 & 3 & 21.4 \\
\hline Irritability & 10 & 0.24 & 4 & 1.7 & - & - \\
\hline Urgent referral note & 135 & 3.52 & 85 & 36.9 & - & - \\
\hline Age $<2$ months & 386 & 10.06 & 73 & 31.7 & - & - \\
\hline ETAT group 1 and/or 2 & 731 & 19.05 & 197 & 85.65 & 14 & 100 \\
\hline No emergency condition or priority signs & 3082 & 80.3 & 33 & 14.3 & - & - \\
\hline Total & 3837 & 100 & 230 & 100 & 14 & 100 \\
\hline
\end{tabular}

${ }^{\star}$ Only deaths within 24 hours from admission are included.

†Patients with group 2 signs only (excluding those with concomitant group 1 classification).

infections). As a whole, the nurses identified 755 patients as having an emergency condition or a priority sign.

APLS assessment used by the paediatricians identified 426 emergency or priority patients. A further 42 patients were identified as needing treatment or close monitoring on the basis of signs not included in the APLS algorithm. A total of 468 patients were identified by the paediatricians as needing priority assessment and treatment.

Two hundred and thirty patients $(5.99 \%$ of patients attending the emergency department) were ultimately admitted to the wards.

There were 14 deaths within 24 hours from admission, two occurring in the emergency department before admission.

The distribution of ETAT signs and classifications, admission, and mortality rates of the various ETAT emergency conditions and priority signs are presented in table 1 .

PERFORMANCE OF ETAT ALGORITHM AS A

SCREENING TOOL

Sensitivity, specificity, and predictive values for the ETAT algorithm as applied by nurses with respect to the reference standards are shown (with 95\% CIs) in table 2. Sensitivity of the ETAT algorithm as applied by nurses was $96.7 \%$ with respect to a positive APLS assessment, $91.7 \%$ with respect to all priority patients, and $85.7 \%$ with respect to patients who were ultimately admitted. Specificity was $90.6 \%, 91.0 \%$, and $85.2 \%$, respectively.

The ETAT algorithm was also compared with the APLS guidelines with respect to the identification of patients who were ultimately admitted (table 3). Sensitivity was $85.7 \%$ for ETAT and $83.0 \%$ for APLS, and specificity was $85.2 \%$ for ETAT and $93.5 \%$ for APLS.
There were 294 young infants with no emergency or priority sign other than age less than 2 months. Twenty two of them were admitted $(9.5 \%$ of all admissions, positive predictive value $10.59 \%) ; 20 / 22$ of these infants showed other signs (such as purulent eye discharge, pyodermatitis, strangulated inguinal hernia, diarrhoea with moderate dehydration) that, although not included in the ETAT algorithm, were recognised by the nurse as signs requiring priority care.

Performance of ETAT using a lower age threshold (infants aged less than 1 month) as a priority sign with respect to admitted patients is shown in table 3 .

AGREEMENT BETWEEN NURSES AND

PAEDIATRICIANS IN USING THE ETAT ALGORITHM For emergency conditions, comparing with the ETAT assessment made by the paediatrician, the nurses failed to identify four children (false negatives): two with severe respiratory distress, one with shock, and one with severe dehydration. They identified wrongly three cases as needing emergency treatment (false positives): one child with only moderate dehydration and two with only moderate respiratory distress. Overall agreement $(\kappa)$ between nurses and doctors in use of ETAT for emergency (group 1) conditions was 0.96 .

For priority signs, there was complete concordance on identification of severe pallor and oedema of the feet, while nurses identified irritability in 10 out of the 16 cases identified by doctors, and two out of three cases of lethargy. Nurses identified one case of severe marasmus not confirmed by a doctor. Nurses identified 213 cases of visible respiratory distress through examination only, while doctors identified 226 cases through observation and auscultation. Overall agreement $(\kappa)$ for severe

Table 2 Sensitivity, specificity, and predictive values (with 95\% CI) of overall ETAT assessment with respect to APLS assessment, all priority cases identified by paediatricians, and all admitted patients

\begin{tabular}{|c|c|c|c|c|c|c|}
\hline Reference standard & $\begin{array}{l}\text { Number of patients } \\
\text { (\% of total) }\end{array}$ & $\begin{array}{l}\text { Number correctly } \\
\text { identified by } \\
\text { ETAT }\end{array}$ & Sensitivity (\%) & Specificity (\%) & $\begin{array}{l}\text { Positive predictive } \\
\text { values }\end{array}$ & $\begin{array}{l}\text { Negative predictive } \\
\text { values }\end{array}$ \\
\hline $\begin{array}{l}\text { Patients positive on APLS assessment } \\
\text { All priority patients as identified by }\end{array}$ & $426 / 3837(11)$ & 412 & 96.7 (94.4 to 98.1$)$ & $90.6(89.6$ to 91.6$)$ & $56.4(52.7$ to 60.0$)$ & $99.5(99.2$ to 99.7$)$ \\
\hline paediatricians & $468 / 3837(12)$ & 429 & 91.7 (88.7 to 93.9$)$ & $91.0(90.0$ to 92.0$)$ & $58.7(55.0$ to 62.3$)$ & 98.7 (98.3 to 99.1$)$ \\
\hline All admitted patients & $230 / 3837(6)$ & 197 & 85.7 (80.3 to 89.8$)$ & $85.2(84.0$ to 86.3$)$ & 26.9 (23.8 to 30.4$)$ & 98.9 (98.5 to 99.3$)$ \\
\hline
\end{tabular}


Table 3 Comparison of the performance of three algorithms in identifying severely ill children requiring hopital admission

\begin{tabular}{llllll}
\hline $\begin{array}{l}\text { Assessment } \\
\text { method }\end{array}$ & $\begin{array}{l}\text { Number } \\
\text { admitted }\end{array}$ & Sensitivity (\%) & Specificity (\%) & $\begin{array}{l}\text { Positive predictive } \\
\text { value }\end{array}$ & $\begin{array}{l}\text { Negative predictive } \\
\text { value }\end{array}$ \\
\hline APLS & $191 / 230$ & $83.0(77.4$ to 87.5$)$ & $93.5(92.6$ to 94.3$)$ & $44.8(40.1$ to 49.7$)$ & $98.9(98.4$ to 99.2$)$ \\
ETAT & $197 / 230$ & $85.7(80.3$ to 89.8$)$ & $85.2(84.0$ to 86.3$)$ & $26.9(23.8$ to 30.4) & $98.9(98.5$ to 99.3$)$ \\
ETAT $^{\star}$ & $189 / 230$ & $82.2(76.5$ to 86.8$)$ & $89.2(88.1$ to 90.2$)$ & $32.6(28.9$ to 36.7$)$ & $98.7(98.3$ to 99.1$)$ \\
\hline
\end{tabular}

*With only infants $<1$ month as priority sign.

marasmus, oedema, pallor, irritability, and lethargy was 0.94. Among those with severe marasmus and lethargy not recognised by the nurses, one was admitted (with lethargy). Among children with respiratory distress who were ultimately admitted, three were not identified by the nurses.

All children missed by nurses as ETAT group 1 patients were identified by them as ETAT group 2 patients.

PERFORMANCE OF ETAT TREATMENT GUIDELINES Nurses using ETAT guidelines identified 98 group 1 patients with 105 conditions requiring immediate treatment (five children having two conditions, and one child having three). Excluding three patients for whom nurses' classification was not confirmed by the doctor (false positive emergency patients), nurses administered treatment for 102 emergency conditions. This treatment was appropriate in $94 / 102$ cases $(92.2 \%)$, and inappropriate or partially inappropriate in eight cases. The treatment administered by nurses was appropriate also in the four false negative emergency conditions after the appropriate assessment was made by the doctor, for a total of $98 / 106$ conditions $(92.5 \%)$ in which the treatment was correctly administered. Reasons for inappropriate treatment were: severe respiratory distress-airway management not done or inadequate (two cases); oxygen not given and patient only prescribed nebulised salbutamol (one case); shock in a patient who arrived at the emergency department with 5\% dextrose intravenous solution-no switch to appropriate intravenous fluid (one case); severe respiratory distress/severe dehydration/shock-fluids not administered (one case); convulsions-no airway management and no blood glucose check (one case); convulsions-no blood glucose check (two cases).

The time necessary to administer treatment was also recorded for each specific treatment. On one occasion the time taken by the nurse to administer treatment (inserting an intravenous line) was judged excessive, provoking direct intervention by the paediatrician. Advanced resuscitation procedures including endotracheal intubation were needed in three cases, including the two patients who eventually died in the emergency department. These were performed by the paediatrician.

The performance of the nurses in delivering emergency treatment improved from 50/56 emergency conditions appropriately treated $(89 \%)$ in the first five weeks of the study to $48 / 50(96 \%)$ in the second half of the study period.

\section{Discussion}

This study was devised to validate the ETAT assessment algorithm and treatment guidelines as used by nurses in a setting that presented many of the epidemiological and organisational characteristics of paediatric referral care in developing countries. Although there are a number of studies evaluating the predictive value of individual signs and symptoms with respect to death or admission, ${ }^{4-7}$ this is the first study which evaluates an emergency algorithm as a whole in comparison to an accepted international standard for emergency care. A large referral hospital was chosen as the study site to allow a sufficient case load. The ETAT guidelines, used by nurses after 20 hours of intensive training, performed well both as a screening tool to identify emergency and priority cases, and as a guide to emergency treatment. The study found that $92.2 \%$ of patients requiring emergency treatment according to the algorithm received adequate and prompt treatment by nurses and the paediatrician had to intervene in only 8/105 cases. There was close concordance between nurses and paediatricians in assessing and classifying priority signs and emergency conditions and it compared well with previous studies evaluating the concordance between doctors and other health workers after training. ${ }^{89}$

The referral hospital has a high patient load, thus providing opportunities for continuous practice of the guidelines. The study period was in "winter" with a higher prevalence of respiratory than diarrhoeal diseases (although seasonal differences are not great in Recife). There were no cases of severe malaria. The professional background of the six nurses involved in the study included high school diploma and three to four years of specific nurse training. These features should be kept in mind when considering the generalisability of our study results in other settings.

One objective was to evaluate the proportion of paediatric patients attending an accident and emergency department with one or more signs indicating the need for emergency treatment or priority assessment and treatment, since if the ETAT algorithm is applicable to only a very small proportion of cases the benefit would be small and compliance poor or difficult to maintain. Conversely, the proportion of children triaged as emergency or priority cases must not be too high, to avoid excessive demand on heavily burdened health professionals. In our study $1 / 40$ cases required emergency treatment and $1 / 6$ required priority assessment. These proportions appear compatible with optimal use of scarce resources and with the ability to maintain high quality care. 
We adopted several reference standards, in an attempt to provide a wider array of comparisons. The main standard was patients identified by an APLS assessment made by expert paediatricians. However, the APLS and ETAT classifications are not fully comparable. ${ }^{2}$ Therefore, all patients identified by paediatricians as needing emergency or priority management (APLS assessment plus any adjunctive priority criteria based on standard medical assessment) were also used as a reference standard. Admitted patients were chosen as the best proxy for an outcome variable. This was reasonable as there are strict admission rules at IMIP, owing to a shortage of beds and other resources, and children are not admitted unless severely ill or at high risk for severe disease. Deaths were too rare to be used as a reference variable. The study was not designed to evaluate any change in mortality rate consequent to the improved emergency care.

Our data show that a lower age threshold for young infants (less than 1 month) would allow a gain in specificity (from $85.2 \%$ to $89.2 \%$ ) with a minor loss in sensitivity (from $85.7 \%$ to $82.2 \%$ ) in identifying those who need priority care for possible serious illness. Excluding the age criterion would have led to missing 22 patients who were ultimately considered severely ill enough to be admitted, although nurses, based on their own clinical judgement, were able to identify 20 out of these as needing priority assessment. Further evaluation of the triage of sick young infants is required.

The duration of training (20 hours) appears compatible with large scale implementation, although a longer training period may be necessary for less skilled health workers. ${ }^{9}{ }^{10}$

Further studies are necessary to evaluate the extent to which the results obtained in Recife can be reproduced in different settings, includ- ing countries with malaria and using less skilled workers, and to estimate how much the implementation of the ETAT guidelines could reduce hospital mortality in developing countries.

We thank the IMIP for enthusiastic support, and Adriana Gomes do Monte, Giorgia da Cunha Cruz, Roberta Campos Siqueira, Jane Schutz Bienik, Helenluci Mendes Francisco, Epifania Leite de Figueiredo, and Edilani Lima Melo for their commitment to the successful completion of the study. The study was supported by the WHO division of child health and development, Geneva.

1 Gove S, for the WHO Working Group on Integrated Management of Childhood Illness. Integrated management of childhood illness by outpatient health workers: technical
basis and overview. Bull World Health Organ,1997;75(suppl basis and

2 Gove S, Tamburlini G, Molineux L, Whitesell P, Campbelle $\mathrm{H}$, for the WHO-IMCI Referral Care Project. Development and technical basis of simplified guidelines for emergency triage assessment and treatment in developing countries Arch Dis Child 1999;81:473-7.

3 Advanced Life Support Group. Advanced paediatric life support - the practical approach, 2nd ed. London: BMJ Pubishing Group, 1997.

4 Campbell H, Byass P, Lamont AC, et al. Assessment of clinical criteria for identification of severe acute lower respiratory tract infections in children. Lancet 1989;i:297-9.

5 Paxton LA, Redd SC, Steketee RW, Otieno JO, Nahlen B. An evaluation of clinical indicators for severe paediatric illness. Bull World Health Organ 1996;74:613-18.

6 Weber MW, Kellingray SD, Palmer A, Jaffar S, Mulholland EK, Greenwood BM. Pallor as a clinical sign of severe anemia in children: an investigation in the Gambia. Bull World Health Organ 1997;75(suppl 1):113-18.

7 Bern C, Zucker JR, Perkins BA, et al. Assessment of potenBern C, Zucker JR, Perkins BA, et al. Assessment of poten-
tial indicators for protein-energy malnutrition in the algorithm for integrated management of childhood illness. Bull World Health Organ, 1997;75(suppl 1):87-96.

8 Kalter HD, Schillinger JA, Hosain M, et al. Identifying sick children requiring referral to hospital in Bangladesh. Bull World Health Organ 1997;75(suppl 1);65-75.

9 Simoes EAF, Desta T, Tessema T, Gerbreselassie T, Dagnew M, Gove S. Performance of health workers after training in integrated management of childhood illness in Gondar, Ethiopia. Bull World Health Organ 1997;75(suppl 1):43-53.

10 WHO Division of Child Health and Development and WHO Regional Office for Africa. Integrated management of childhood illness: field test of the WHO-UNICEF training course in Arusha, United Republic of Tanzania. Bull World Health Organ 1997;75(suppl 1):55-64. 